\title{
Erratum: Temperature Dependence of the Nitrogen-Vacancy Magnetic Resonance in Diamond [Phys. Rev. Lett. 104, 070801 (2010)]
}

\author{
V. M. Acosta, ${ }^{*}$ E. Bauch, M. P. Ledbetter, A. Waxman, L.-S. Bouchard, and D. Budker \\ *victor.acosta@hp.com \\ (Received 15 April 2011; published 16 May 2011) \\ DOI: 10.1103/PhysRevLett.106.209901 \\ PACS numbers: 07.55.Jg, 76.30.Mi, 85.70.Sq, 99.10.Cd
}

In the Letter we reported a study of the temperature dependence of the magnetic-resonance spectra of nitrogen-vacancy (NV) centers in diamond near room temperature. Our main conclusion was that, in the 280-330 K range, the axial zerofield splitting parameter $D$ has a significant temperature dependence which presents an important challenge for magnetometry applications. This conclusion remains unchanged. However, an error in our fitting procedure led to some mistakes in the analysis that we have corrected here.

In Fig. 1 of the Letter we fit to incorrect transition frequencies, which led to errors in the fitted values of $E$ (the transverse zero-field splitting parameter) and $D$. The correct fit is shown here in Fig. 1. We reanalyzed all of the data in the Letter and found that most of the $E(D)$ values in the original Letter were too large (small) by 1-3 MHz. Table I shows the results of the reanalysis (compare to Table I in the original Letter).

The reanalysis confirmed all of the conclusions in the original Letter. Table II compares the temperature dependence of $D$ and $E$ obtained in the original Letter and after the reanalysis. The values are identical to within their uncertainties.

The authors gratefully acknowledge L. J. Zipp and A. Jarmola for essential contributions to this erratum.

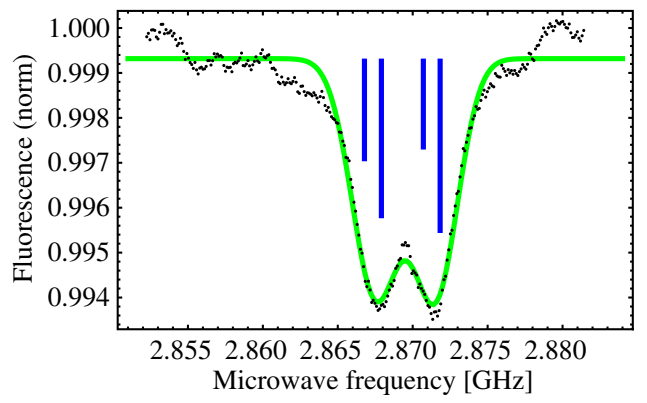

FIG. 1 (color online). Fit to the data in Fig. 1 of the Letter using the corrected analysis. There are only four nondegenerate microwave transitions (solid blue lines), and they are positioned symmetric about the axial zero-field splitting parameter $D$. Other smaller features were not reproduced in subsequent measurements and are possibly due to noise from the acquisition electronics. From the fit, we find $E=1.4(5) \mathrm{MHz}$ and $D=2869.3(2) \mathrm{MHz}$ for this sample, $S 3$.

TABLE I. Results of the reanalysis for each of the four samples. The main differences between this table and Table I in the original Letter are the values of $E$.

\begin{tabular}{lcccc}
\hline \hline No. & {$\left[\mathrm{NV}^{-}\right](\mathrm{ppm})$} & $\frac{d D}{d T}(\mathrm{kHz} / \mathrm{K})$ & $E(\mathrm{MHz})$ & $\frac{1}{E} \frac{d E}{d T}\left(10^{-4} \mathrm{~K}^{-1}\right)$ \\
\hline$S 2$ & 16 & $-71(1)$ & $2.7(6)$ & $-5(2)$ \\
S3 & 0.01 & $-74(4)$ & $1.4(5)$ & $-40(40)$ \\
S5 & 12 & $-78(1)$ & $8(1)$ & $-5(1)$ \\
S8 & 0.3 & $-76(1)$ & $2.6(4)$ & $-0.4(5)$ \\
\hline \hline
\end{tabular}

TABLE II. The fractional temperature dependence of the zero-field splitting parameters, which were the main results in the Letter, remain unchanged to within their uncertainty.

\begin{tabular}{lccc}
\hline \hline & $\frac{d D}{d T}(\mathrm{kHz} / \mathrm{K})$ & $\frac{1}{D} \frac{d D}{d T}\left(10^{-5} \mathrm{~K}^{-1}\right)$ & $\frac{1}{E} \frac{d E}{d T}\left(10^{-4} \mathrm{~K}^{-1}\right)$ \\
\hline Letter & $-74.2(7)$ & $-2.59(2)$ & $-1.4(3)$ \\
Corrected & $-75.0(6)$ & $-2.61(2)$ & $-1.5(5)$ \\
\hline \hline
\end{tabular}

